\title{
The fibrotic microenvironment as a heterogeneity facet of hepatocellular carcinoma
}

\author{
Krista Rombouts ${ }^{1}$ and Vinicio Carloni ${ }^{2^{*}}$
}

\begin{abstract}
It has long been recognized that hepatocellular carcinoma heterogeneity arises from variation in the microenvironment or from genomic alteration. Only recently it has become clear that non-genetic alterations, such as cytoskeletal rearrangement, protein localization and formation of protein complexes, are also involved in generating phenotype variability. These proteome fluctuations cause genetically identical cells to vary significantly in their responsiveness to microenvironment stimuli. In the cirrhotic liver pre-malignant hepatocytes are continuously exposed to abnormal microenvironments, such as direct contact with activated hepatic stellate cells (HSCs) and extracellular matrix components. These abnormal environments can have pronounced influences on the epigenetic aspects of cells, translating into abnormal phenotypes. Here we discuss non-genetic causes of phenotypic heterogeneity of hepatocellular carcinoma, with an emphasis on variability of membrane protein complexes and transferred functions raising important implications for diagnosis and treatment.
\end{abstract}

Keywords: Liver cancer, Activated hepatic stellate cells (HSC), Intra-tumor heterogeneity, Tetraspanin-enriched microdomains, Integrins

\section{Review}

Introduction

Hepatocellular carcinoma (HCC) is the third leading cause of cancer mortality worldwide and a significant increase in the incidence of HCC through the last two decades has been observed [1]. There are two prominent features in the development of HCC. First, 90\% of HCCs have chromosomal abnormalities and, second, the great majority of these tumors, regardless of aetiology, develop in cirrhotic livers, which are characterized by destruction of the hepatic lobular architecture and its replacement by nodules containing proliferative hepatocytes, in the presence of chronic inflammation and fibrosis [2].

A seminal feature of hepatocellular carcinoma is the ability to produce multiple subpopulations of cells with diverse genetic, biochemical and immunological characteristics $[3,4]$. How this heterogeneity emerges and how it is maintained is not clear $[5,6]$. Fluctuations in single cells can be masked or completely misrepresented when cell populations are analyzed. Therefore, intra-tumor

\footnotetext{
*Correspondence: vinicio.carloni@unifi.it

${ }^{2}$ Department of Experimental and Clinical Medicine, Center for Research, Transfer and High Education, DENOthe, University of Florence, Largo Brambilla 3, 50134, Florence, Italy

Full list of author information is available at the end of the article
}

heterogeneity may foster tumor evolution and adaptation and hinder personalized-medicine strategies that depend on results from imaging procedures or single tumor-biopsy samples $[7,8]$. Along these lines, it has become exceedingly apparent that the utility of measurements based on the analysis of bulk tumors is limited by intra-tumor genetic and epigenetic heterogeneity, as characteristics of the most abundant cell type might not necessarily predict the properties of the whole cell populations [8]. Indeed, this aspect is supported by a recent report describing the presence of distinct diagnostic signatures derived from different biopsies of the same tumor [9]. Yet, such non-uniformities often unveil molecular patterns that can represent mechanisms of tumor progression. More interestingly, variability among single cells in a population may arise from different responses to intrinsic and extrinsic perturbations coming from the abnormal microenvironment that may have pronounced influences on the epigenetic aspects of cells, translating into abnormal phenotypes [10]. Therefore, it is tantalizing to hypothesize that normalization of the tumor microenvironment corresponds to the normalization of cellular phenotypes, and destabilization of normal tissue organization can translate into an increased risk of 
genomic instability and phenotype heterogeneity $[11,12]$. The great interest concerning the tumor microenvironment is associated with the recognition that micro-environ mental alterations are not just passive consequences of genetic evolution occurring in hepatocytes, but that they are active participants in tumorigenesis [13]. As many excellent reviews summarize progress in this area, we focus on the effects of micro-environmental alterations on the phenotypic heterogeneity of pre-malignant hepatocytes.

\section{Activated hepatic stellate cells}

Pre-malignant hepatocytes live in a complex microenvironment that includes the extracellular matrix (ECM), diffusible growth factors and cytokines, and a variety of non-epithelial cell types, including endothelial cells, activated hepatic stellate cells (HSCs), and those that can respond to infection and injury, that is, lymphocytes, Kupffer cells-macrophages and mast cells $[14,15]$.

HSCs are known as very important ECM-producing myofibroblasts dwelling in the cirrhotic liver and microenvironment of HCC. The activated HSCs infiltrate the stroma of liver tumors and localize around tumor sinusoids, fibrous septa and capsules [16,17]. Activated HSCs increase the production and secretion of ECM proteins, which include collagens, laminins, fibronectin and heparan-sulphate proteoglycans. In this way, HSCs have a major impact on the ECM content of the microenvironment and also may affect the overall tumor stromal behavior and vice versa [18]. Indeed, several studies demonstrated that transformed hepatocytes stimulate migration of HSCs in culture, as well as their production of ECM components, when co-cultured, or when HCC tumor-conditioned medium was used [19-21]. Hence, all support the concept that hepatocarcinoma cells recruit HSCs, which then promote tumor growth and local invasion [22]. The cancer cell-induced increase in ECM synthesis is mediated by transforming growth factor beta (TGF- $\beta 1$ ), whereas proliferation of HSCs is promoted by platelet-derived growth factor (PDGF) [23,24]. This interaction, between HCC and HSCs, is bidirectional since HSCs, in turn, stimulate hepatocarcinoma cell proliferation and inhibit their apoptosis to increase the population of cancer cells [25]. Proliferation of hepatocytes is mediated by factors secreted by activated HSCs, such as insulin-like growth factor I (IGF-I), transforming growth factor alpha (TGF- $\alpha$ ), hepatocyte growth factor (HGF) and other inflammatory cytokines [26,27]. Accumulating evidence indicates and points to an important and major influence of activated HSC on HCC development and progression and, hence, the therapeutic inhibition of activated HSCs should be taken into account when treating HCC [28].

\section{Premalignant and cancerous hepatocytes Hepatocyte plasma membrane microdomains, the tetraspanin paradigm}

It has long been recognized that differences from one cell to the next can arise through variation in the extracellular environment or from genomic alteration. Only recently it has become clear that plasma membrane protein fluctuations can also have profound effects on phenotype. These fluctuations cause genetically identical cells to vary significantly in their responsiveness to stimuli of the fibrotic microenvironment (Figure 1).

The spatial organization of plasma membrane components in discrete microdomains is thought to be a key factor in the generation of distinct signal inputs or outputs [29]. Dynamic microdomains have important implications for understanding how signaling complexes are assembled and disassembled in response to ECM stimuli; some components of these signaling complexes might reside permanently in these microdomains, but others could have extremely transient interactions [30].

Tetraspanins are transmembrane proteins defined by small and large outer loops, short N-terminal and Cterminal tails with four transmembrane domains. They form complexes termed tetraspanin-enriched microdomains (TEMs) by interacting with other tetraspanins and with a variety of transmembrane and cytosolic proteins that are required for their function [31]. Several tetraspanin molecules have been identified and implicated in the regulation of cell proliferation, cell migration and cell fusion [32]. The most important partners are integrins, particularly $\alpha 3 \beta 1, \alpha 4 \beta 1, \alpha 6 \beta 1$ and $\alpha 6 \beta 4$, intracellular associated heterotrimeric $\mathrm{G}$ proteins, proteases, immunoglobulin superfamily members and cytosolic signal transduction molecules [31]. The repertoire of tetraspanins differs between cancer cell types; therefore, a complete characterization of tetraspanin-associated proteins and functions is difficult to accomplish and may not be generalized. Nevertheless, in the majority of cancer cell types, including HCC, a characteristic feature is the evident presence of integrins, signaling proteins and proteases as important components of these domains [33].

\section{Tetraspanin CD81}

Tetraspanin CD81 was identified originally as the target of an anti-proliferative antibody (TAPA-1) that inhibited in vitro cellular proliferation [34]. CD81 is involved in a broad range of cellular functions as revealed by the binding of monoclonal antibodies. The antibodies evoke their effect by mimicking a natural ligand or by altering the interactions between CD81 and its associated proteins. Although the protein is widely expressed, its levels within a single tissue vary in response to cellular activation. An important feature of tetraspanin CD81 is its ability to associate with itself forming homodimers and with various other 


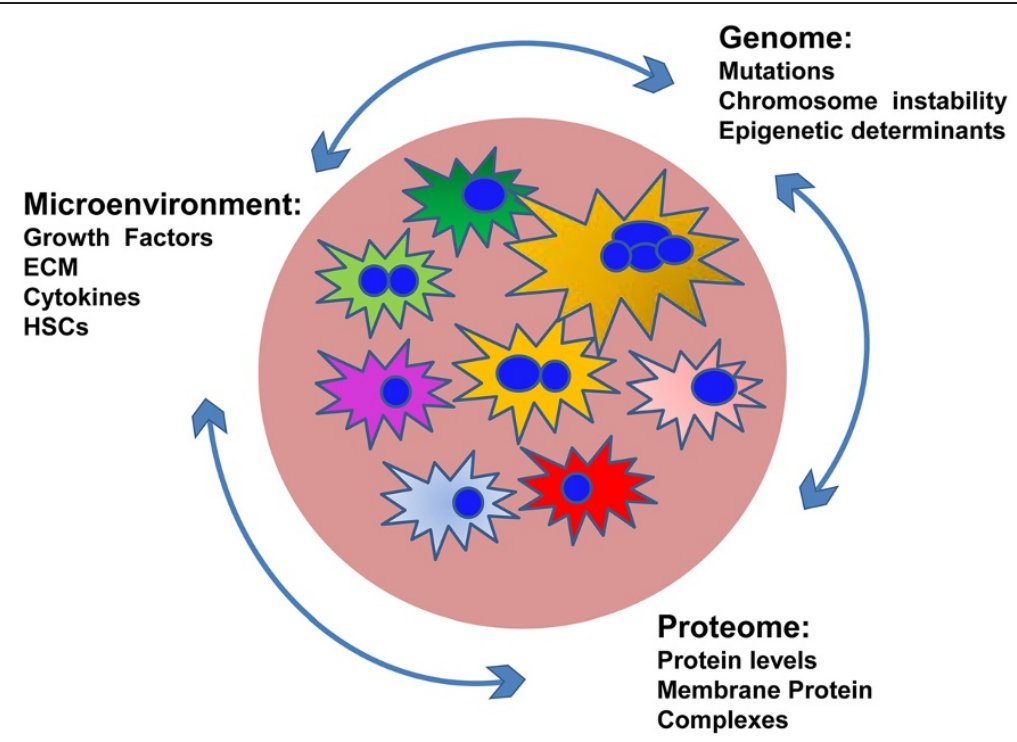

Figure 1 Interaction among factors that determine phenotypic heterogeneity in HCC. Combinations of environmental, genomic and proteomic variation can cause heterogeneity in an initially homogenous population of pre-malignant hepatocytes.

receptors into membrane microdomains. Up-regulation of CD81 in pre-malignant hepatocytes can contribute to reorganizing the plasma membrane in domains where signaling proteins can be recruited [35]. CD81 regulation of proliferation is positively associated with activation of the extracellular signal-regulated kinase $1 / 2$ (ERK1/2)/MAPK pathway. CD81 overexpression can activate ERK1/2 while promoting proliferation [35]. Importantly, CD81 induces reorganization of the plasma membrane amplifying the instability of pre-malignant hepatocytes and enhancing their neoplastic progression. Therefore, phenotype heterogeneity could be influenced primarily by a fluctuation of a single protein and associated factors organized in discrete plasma membrane domains. These membrane microdomains represent versatile devices for compartmentalizing different signaling functions. In the non-activated state they float freely, carrying a few passenger proteins, but, when activated, they coalesce to form larger platforms where proteins meet to transfer functions in signaling, processing and transport [36].

When the hepatocytes progress in their transformation, the tendency of CD81 expression is to be lost, as revealed by two clinical studies showing a decreased or absent CD81 expression, particularly in metastatic tissues $[37,38]$. HCC cells re-expressing CD81 are still capable of proliferating and producing the principal tumor when injected into the liver of nude rats; however, they contain a defective faculty to produce tumors in distant parts of the liver [39]. These findings strengthen the vision that CD81 is a facilitator of cell proliferation and in the meantime is a negative controller/regulator of movement when expressed by the cells. This is supported by the current view that cell growth and ability to metastasize are two conditions of malignancy not necessarily overlapping [40].

\section{Tetraspanin CD151}

The initial evidence that CD151 promotes metastasis came from a study showing that an antibody with unknown specificity inhibited metastasis formation by a human epidermoid carcinoma cell line in vivo. The antibody was found to recognize CD151, and inhibit cell migration without affecting adhesion or proliferation [41]. Overexpression of CD151 is seen in many tumor types. In breast, pancreatic, colorectal and non-smallcell lung cancer, high CD151 expression is associated with a poor prognosis [33].

Overexpression of CD151 has been associated with poor prognosis also in HCC. Some studies have indicated that CD151 overexpression promotes the metastasis/invasion of cancer cells by mediating integrin signals, while others have argued that an increased expression of CD151 contributes to activate phosphatidylinositol 3kinase/protein kinase Akt pathway [42]. Indeed, the high expressions of CD151 and $\alpha 6$ integrin are major contributors to the invasion-prone phenotype of HCC. In contrast with CD81, the contribution of CD151 to HCC metastasis/invasion provides an example of the facilitator role of this tetraspanin (Figure 2). Apart from CD151, the tetraspanin TSPAN8 (previously known as CO-029, TM4SF3) has been also associated with tumor progression [43]. Overexpression of TSPAN8 is described on hepatocellular carcinomas that are poorly differentiated and prone to intrahepatic spreading [44]. Conversely, down-regulation of tetraspanin CD82/KAI1 was observed at the levels of both mRNA and protein. 


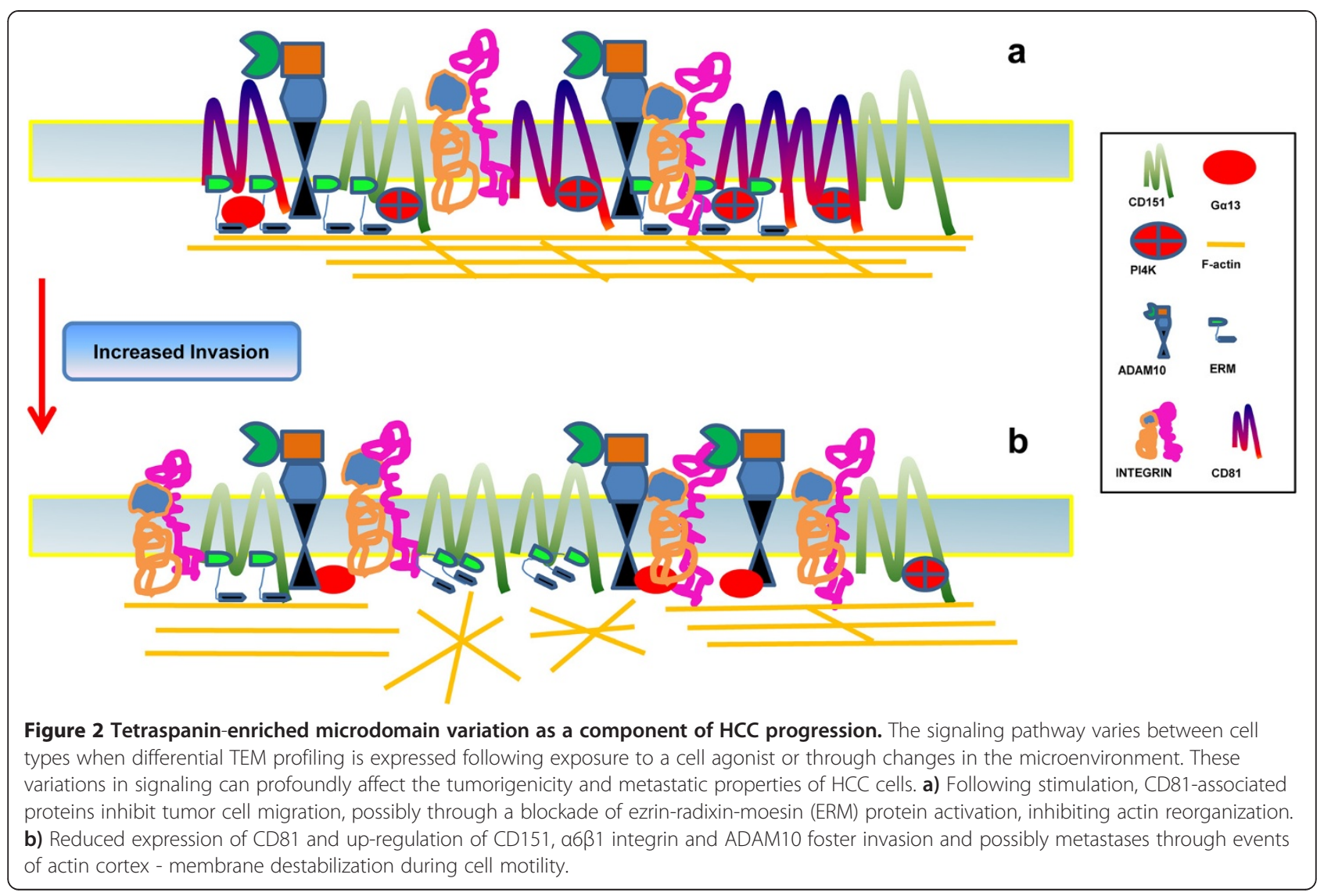

This was particularly pronounced in poorly differentiated HCCs. Importantly, the CD82/KAI1 level correlated inversely with intrahepatic metastases [45].

\section{Differences in integrin expression and signaling within HCC}

The pattern of integrins expressed by human hepatocytes is strikingly different from most other epithelial cells [46]. Normal adult hepatocytes express low levels of only three integrins: $\alpha 1 \beta 1$, a collagen and laminin receptor; $\alpha 5 \beta 1$, a fibronectin receptor; and $\alpha 9 \beta 1$, a tenascin receptor. In contrast, other integrin receptors, such as $\alpha 2 \beta 1, \alpha 3 \beta 1$, $\alpha 6 \beta 1$ and $\alpha 6 \beta 4$, are undetectable on normal hepatocytes. One of the most frequent alterations during liver carcinogenesis is the de novo expression of the integrin, $\alpha 6 \beta 1$. HCC patients characterized by multiple tumors, vascular invasion and the absence of encapsulation exhibit increased $\alpha 6 \beta 1$ expression [47]. In fact, the induction of $\alpha 6 \beta 1$ is an early event in hepatocellular carcinogenesis, and it is reasonable to consider that $\alpha 6 \beta 1$ contributes to hepatocarcinogenesis based on several lines of evidence [48-51]. For this reason, it is important to understand the mechanism by which the $\alpha 6 \beta 1$ integrin influences the function of HCC cells. One likely possibility is that $\alpha 6 \beta 1$ mediated activation of focal adhesion kinase (FAK) and ERK1/2 controls signaling pathways important for HCC function [52]. Both FAK and ERK1/2 are of interest because they are regulated by integrin-mediated attachment to ECM, as well as growth factor stimulation, and they control important functions of tumor cells, such as growth and migration. Another possibility suggests that overexpression of $\alpha 6 \beta 1$ could provide a ligand-independent growth advantage by modulating the cellular architecture or a signaling pathway required for cell growth [51].

Interestingly, the role of $\alpha 3 \beta 1$ integrin appears more controversial in hepatocarcinogenesis. A previous study indicated that TGF- $\beta 1$ was able to induce a significant increase in the expression level of $\alpha 3 \beta 1$, which consecutively cooperated with TGF- $\beta 1$ to induce HCC cell epithelial-mesenchymal transition (EMT) [53]. In a recent study, investigators could not confirm the previous findings when evaluating $\alpha 3 \beta 1$ expression in HCC tissue specimen of patients with high concentration of serum TGF- $\beta 1$ levels nor could be demonstrated a significant up-regulation of $\alpha 3 \beta 1$ in HCC cells after 24 or 48 hours of TGF- $\beta 1$ stimulation. Indeed, they find that the amplified integrin $\alpha 6 \beta 1$ signaling pathway is able to induce EMT of HCC cells [42].

\section{ADAMs}

ADAMs are multidomain proteins that contain a disintegrin and a metalloprotease domain [54]. Their metalloprotease domains can induce ectodomain shedding and cleave ECM 
proteins [55]. Their disintegrin and cysteine-rich domains have adhesive and fusion activities. Hence, ADAMs are poised to modulate a variety of cell-cell and cell-ECM interactions. ADAM10, a member of the ADAM family, was detected in all human HCC tissues tested by immunohistochemistry but not in normal liver tissues [56]. Moreover, CD44, a typical substrate of ADAM10 protease, was also expressed in all human HCC tissues but not in normal liver tissues. These data suggest that overexpression of ADAM10 and CD44 is a characteristic of human HCC. Specifically, ADAM10 is involved in the intramembrane proteolysis process, whereby it mediates ectodomain shedding of various membrane-bound receptors, adhesion molecules, growth factors and cytokines [57].

\section{Conclusions}

HCC cell phenotypes are the result of the integration of inputs from genotype and environmental stimuli. Epigenetic changes that arise during tumor progression alter and diversify cellular phenotypes, posing a major obstacle to the understanding and clinical management of HCC. We suggest that the phenomenon of intratumor phenotypic heterogeneity, especially aspects that are related to clonal diversity, deserve to be recognized and accounted for during the analysis of HCC tumor, building of experimental models and design of therapeutic approaches.

The dominance of gene-centric views has been challenged with the rapid development of research establishing that because tumors contain phenotypically distinct populations of both tumor and stromal cells that interact in a dynamic and reciprocal manner, these interactions are likely to result in the emergence of different proteome profiling. This aspect creates significant problems in employing therapeutic procedures in which microenvironmental changes make a procedure inefficient and in some regions of the HCC a therapeutic result may not be achieved. This inequality of therapy gives HCC cells time to develop resistant phenotypes. In addition, components of the microenvironment can actively protect tumor cells from treatment through secreted factors and cell contact-mediated pro-survival stimuli. Heterogeneity in the tumor microenvironment translates into heterogeneity of tumor cell phenotypes, and so some tumor cells might be intrinsically less sensitive to the therapy. Intra-tumor heterogeneity, associated with heterogeneous protein function, may promote HCC progression through Darwinian selection.

\section{Abbreviations}

ADAM: A-disintegrin and metalloprotease; Akt: Protein kinase B;

ECM: Extracellular matrix; EMT: Epithelial-mesenchymal transition; ERK: Extracellular regulated protein kinase; ERM: ezrin-radixin-moesin; FAK: Focal adhesion kinase; HCC: Hepatocellular carcinoma; HGF: Hepatocyte growth factor; HSCs: Hepatic stellate cells; IGF-I: Insulin-like growth factor I; MAPK: Mitogen-activated protein kinase; PDGF: Platelet-derived growth factor; TEMs: Tetraspanin-enriched microdomains; TGF-a: Transforming growth factor-alpha; TGF- $\beta 1$ : Transforming growth factor-beta.

\section{Competing interests}

The authors declare that they have no competing interests.

\section{Author's contributions}

KR and VC wrote the manuscript. VC finalized the manuscript and organized the figures. Both authors read and approved the final manuscript.

\section{Acknowledgments}

We acknowledge support from Ente Cassa di Risparmio di Firenze (VC).

\section{Author details}

${ }^{1}$ Institute for Liver and Digestive Health, Royal Free Hospital, University College London UCL, London, UK. ²Department of Experimental and Clinical Medicine, Center for Research, Transfer and High Education, DENOthe, University of Florence, Largo Brambilla 3, 50134, Florence, Italy.

Received: 23 July 2013 Accepted: 28 August 2013

Published: 16 September 2013

\section{References}

1. El-Serag HB: Hepatocellular carcinoma. N Engl J Med 2011, 365:1118-1127.

2. Farazi PA, DePinho RA: Hepatocellular carcinoma pathogenesis: from genes to environment. Nat Rev Cancer 2006, 6:674-687.

3. Li Y, Tang ZY, Hou JX: Hepatocellular carcinoma: insight from animal models. Nat Rev Gastroenterol Hepatol 2012, 9:32-43.

4. Tao Y, Ruan J, Yeh SH, Lu X, Wang Y, Zhai W, Cai J, Ling S, Gong Q, Chong Z, Qu Z, Li Q, Liu J, Yang J, Zheng C, Zeng C, Wang HY, Zhang J, Wang SH, Hao L, Dong L, Li W, Sun M, Zou W, Yu C, Li C, Liu G, Jiang L, Xu J, Huang $H$, et al: Rapid growth of a hepatocellular carcinoma and the driving mutations revealed by cell-population genetic analysis of whole-genome data. Proc Natl Acad Sci U S A 2011, 108:12042-12047.

5. Gerlinger M, Swanton C: How Darwinian models inform therapeutic failure initiated by clonal heterogeneity in cancer medicine. $\mathrm{Br} J \mathrm{Cancer}$ 2010, 103:1139-1143.

6. Luo J, Solimini NL, Elledge SJ: Principles of cancer therapy: oncogene and non-oncogene addiction. Cell 2009, 136:823-837.

7. Brock $A$, Chang $H$, Huang S: Non-genetic heterogeneity a mutationindependent driving force for the somatic evolution of tumours. Nat Rev Genet 2009, 10:336-342.

8. Marusyk A, Almendro V, Polyak K: Intra-tumour heterogeneity: a looking glass for cancer? Nat Rev Cancer 2012, 12:323-334.

9. Gerlinger M, Rowan AJ, Horswell S, Math M, Larkin J, Endesfelder D, Gronroos E, Pierre Martinez P, Matthews N, Stewart A: Intratumor heterogeneity and branched evolution revealed by multiregion sequencing. N Engl J Med 2012, 366:883-892.

10. Lee AJ, Endesfelder D, Rowan AJ, Walther A, Birkbak NJ, Futreal PA, Downward J, Szallasi Z, Tomlinson IP, Howell M, Kschischo M, Swanton C: Chromosomal instability confers intrinsic multidrug resistance. Cancer Res 2011, 71:1858-1870.

11. Egeblad M, Nakasone ES, Werb Z: Tumors as organs: complex tissues that interface with the entire organism. Dev Cell 2010, 18:884-901.

12. Bissell MJ, Kenny PA, Radisky DC: Microenvironmental regulators of tissue structure and function also regulate tumor induction and progression: the role of extracellular matrix and its degrading enzymes. Cold Spring Harb Symp Quant Biol 2005, 70:343-356.

13. TIsty TD, Coussens LM: Tumor stroma and regulation of cancer development. Annu Rev Pathol 2006, 1:119-150.

14. Leonardi GC, Candido S, Cervello M, Nicolosi D, Raiti F, Travali S, Spandidos DA, Libra M: The tumour microenvironment in hepatocellular carcinoma. Int J Oncol 2012, 40:1733-1747.

15. Wu SD, Ma YS, Fang Y, Liu LL, Fu D, Shen XZ: Role of the microenvironment in hepatocellular carcinoma development and progression. Cancer Treat Rev 2012, 38:218-225.

16. Mikula M, Proell V, Fischer AN, Mikulits W: Activated hepatic stellate cells induce tumor progression of neoplastic hepatocytes in a TGF-beta dependent fashion. J Cell Physiol 2006, 209:560-567.

17. Coulouarn C, Corlu A, Glaise D, Guénon I, Thorgeirsson SS, Clément B: Hepatocyte-stellate cell cross-talk in the liver engenders a permissive 
inflammatory microenvironment that drives progression in hepatocellular carcinoma. Cancer Res 2012, 72:2533-2542.

18. Zhao W, Zhang L, Yin Z, Su W, Ren G, Zhou C, You J, Fan J, Wang X: Activated hepatic stellate cells promote hepatocellular carcinoma development in immunocompetent mice. Int J Cancer 2011, 129:2651-2661.

19. Sancho-Bru P, Juez E, Moreno M, Khurdayan V, Morales-Ruiz M, Colmenero J, Arroyo V, Brenner DA, Ginès P, Bataller R: Hepatocarcinoma cells stimulate the growth, migration and expression of pro-angiogenic genes in human hepatic stellate cells. Liver Int 2010, 30:31-41.

20. Faouzi S, Lepreux S, Bedin C, Dubuisson L, Balabaud C, Bioulac-Sage P, Desmoulière A, Rosenbaum J: Activation of cultured rat hepatic stellate cells by tumoral hepatocytes. Lab Invest 1999, 79:485-493.

21. Xia Y, Chen R, Song Z, Ye S, Sun R, Xue Q, Zhang Z: Gene expression profiles during activation of cultured rat hepatic stellate cells by tumoral hepatocytes and fetal bovine serum. J Cancer Res Clin Oncol 2010, 136:309-321.

22. Garcia MG, Bayo J, Bolontrade MF, Sganga L, Malvicini M, Alaniz L, Aquino JB, Fiore E, Rizzo MM, Rodriguez A, Lorenti A, Andriani O, Podhajcer $\mathrm{O}$, Mazzolini G: Hepatocellular carcinoma cells and their fibrotic microenvironment modulate bone marrow-derived mesenchymal stromal cell migration in vitro and in vivo. Mol Pharm 2011, 8:1538-1548.

23. Campbell JS, Hughes SD, Gilbertson DG, Palmer TE, Holdren MS, Haran AC, Odell MM, Bauer RL, Ren HP, Haugen HS, Yeh MM, Fausto N: Plateletderived growth factor $C$ induces liver fibrosis, steatosis, and hepatocellular carcinoma. Proc Natl Acad Sci U S A 2005, 102:3389-3394.

24. Hou X, Kumar A, Lee C, Wang B, Arjunan P, Dong L, Maminishkis A, Tang Z, Li Y, Zhang F, Zhang SZ, Wardega P, Chakrabarty S, Liu B, Wu Z, Colosi P, Fariss RN, Lennartsson J, Nussenblatt R, Gutkind JS, Cao Y, Li X: PDGF-CC blockade inhibits pathological angiogenesis by acting on multiple cellular and molecular targets. Proc Natl Acad Sci U S A 2010, 107:12216-12221.

25. Nevzorova YA, Hu W, Cubero FJ, Haas U, Freimuth J, Tacke F, Trautwein C, Liedtke C: Overexpression of c-myc in hepatocytes promotes activation of hepatic stellate cells and facilitates the onset of liver fibrosis. Biochim Biophys Acta 1832, 2013:1765-1775.

26. van Zijl F, Mair M, Csiszar A, Schneller D, Zulehner G, Huber H, Eferl R, Beug H, Dolznig H, Mikulits W: Hepatic tumor-stroma crosstalk guides epithelial to mesenchymal transition at the tumor edge. Oncogene 2009, 28:4022-4033.

27. Taura K, De Minicis S, Seki E, Hatano E, Iwaisako K, Osterreicher CH, Kodama Y, Miura K, Ikai I, Uemoto S, Brenner DA: Hepatic stellate cells secrete angiopoietin 1 that induces angiogenesis in liver fibrosis. Gastroenterology 2008, 135:1729-1738.

28. Campbell JS, Johnson MM, Bauer RL, Hudkins KL, Gilbertson DG, Riehle KJ, Yeh MM, Alpers CE, Fausto N: Targeting stromal cells for the treatment of platelet-derived growth factor $\mathrm{C}$-induced hepatocellular carcinogenesis. Differentiation 2007, 75:843-852

29. Simons K, Toomre D: Lipid rafts and signal transduction. Nat Rev Mol Cell Biol 2000, 1:31-39.

30. Gao X, Lowry PR, Zhou X, Depry C, Wei Z, Wong GW, Zhang J: PI3K/Akt signaling requires spatial compartmentalization in plasma membrane microdomains. Proc Natl Acad Sci U S A 2011, 108:14509-14514.

31. Yáñez-Mó M, Barreiro O, Gordon-Alonso M, Sala-Valdés M, Sánchez-Madrid F: Tetraspanin-enriched microdomains: a functional unit in cell plasma membranes. Trends Cell Biol 2009, 19:434-446.

32. Mazzocca A, Carloni V, Sciammetta S, Cordella C, Pantaleo P, Caldini A, Gentilini $P$, Pinzani M: Expression of transmembrane 4 superfamily (TM4SF) proteins and their role in hepatic stellate cell motility and wound healing migration. J Hepatol 2002, 37:322-330.

33. Zoller M: Tetraspanins: push and pull in suppressing and promoting metastasis. Nat Rev Cancer 2009, 9:40-55.

34. Oren R, Takahashi S, Doss C, Levy R, Levy S: TAPA-1, the target of an antiproliferative antibody, defines a new family of transmembrane proteins. Mol Cell Biol 1990, 10:4007-4015.

35. Carloni V, Mazzocca A, Ravichandran KS: Tetraspanin CD81 is linked to ERK/MAPKinase signaling by Shc in liver tumor cells. Oncogene 2004, 23:1566-1574

36. Mukherjee S, Maxfield FR: Membrane domains. Annu Rev Cell Dev Biol 2004, 20:839-866.

37. Inoue $\mathrm{G}$, Horiike N, Onji M: The CD81 expression in liver in hepatocellular carcinoma. Int J Mol Med 2001, 7:67-71.

38. Schöniger-Hekele M, Hänel S, Wrba F, Müller C: Hepatocellular carcinomasurvival and clinical characteristics in relation to various histologic molecular markers in Western patients. Liver Int 2005, 25:62-69.
39. Mazzocca A, Liotta F, Carloni V: Tetraspanin CD81-regulated cell motility plays a critical role in intrahepatic metastasis of hepatocellular carcinoma. Gastroenterology 2008, 135:244-256.

40. Chiang AC, Massague J: Molecular basis of metastasis. N Engl J Med 2008, 359:2814-2823.

41. Testa JE, Brooks PC, Lin JM, Quigley JP: Eukaryotic expression cloning with an antimetastatic monoclonal antibody identifies a tetraspanin (PETA-3/ CD151) as an effector of human tumor cell migration and metastasis. Cancer Res 1999, 59:3812-3820.

42. Ke AW, Shi GM, Zhou J, Huang XY, Shi YH, Ding ZB, Wang XY, Devbhandari RP, Fan J: CD151 amplifies signaling by integrin $a 6 \beta 1$ to PI3K and induces the epithelial-mesenchymal transition in HCC cells. Gastroenterology 2011, 140:1629-1641.

43. Gesierich S, Berezovskiy I, Ryschich E, Zoller M: Systemic induction of the angiogenesis switch by the tetraspanin D6.1A/CO-029. Cancer Res 2006, 66:7083-7094

44. Kanetaka K, Sakamoto M, Yamamoto Y, Takamura M, Kanematsu T, Hirohashi S: Possible involvement of tetraspanin CO-029 in hematogenous intrahepatic metastasis of liver cancer cells. J Gastroenterol Hepatol 2003, 18:1309-1314.

45. Guo XZ, Friess H, Di Mola FF, Heinicke JM, Abou-Shady M, Graber HU, Baer HU, Zimmermann A, Korc M, Büchler MW: KAl1, a new metastasis suppressor gene, is reduced in metastatic hepatocellular carcinoma. Hepatology 1998, 28:1481-1488.

46. Guo W, Giancotti FG: Integrin signalling during tumour progression. Nat Rev Mol Cell Biol 2004, 5:816-826.

47. Begum NA, Mori M, Matsumata T, Takenaka K, Sugimachi K, Barnard GF: Differential display and integrin alpha 6 messenger RNA overexpression in hepatocellular carcinoma. Hepatology 1995, 22:1447-1455.

48. Torimura T, Ueno T, Kin M, Inuzuka S, Sugawara H, Tamaki S, Tsuji R, Sujaku K, Sata M, Tanikawa K: Coordinated expression of integrin alpha6beta1 and laminin in hepatocellular carcinoma. Hum Pathol 1997, 28:1131-1138

49. Wewer UM, Shaw LM, Albrechtsen R, Mercurio AM: The integrin alpha 6 beta 1 promotes the survival of metastatic human breast carcinoma cells in mice. Am J Pathol 1997, 151:1191-1198.

50. Nejjari M, Hafdi Z, Dumortier J, Bringuier AF, Feldmann G, Scoazec JY: alpha6beta1 integrin expression in hepatocarcinoma cells: regulation and role in cell adhesion and migration. Int J Cancer 1999, 83:518-525.

51. Carloni V, Romanelli RG, Mercurio AM, Pinzani M, Laffi G, Cotrozzi G, Gentilini P: Knockout of alpha6 beta1-integrin expression reverses the transformed phenotype of hepatocarcinoma cells. Gastroenterology 1998, 115:433-442

52. Carloni V, Mazzocca A, Pantaleo P, Cordella C, Laffi G, Gentilini P: The integrin, alpha6beta1, is necessary for the matrix-dependent activation of FAK and MAP kinase and the migration of human hepatocarcinoma cells. Hepatology 2001, 34:42-49.

53. Giannelli G, Bergamini C, Fransvea E, Sgarra C, Antonaci S: Laminin-5 with transforming growth factor-beta1 induces epithelial to mesenchymal transition in hepatocellular carcinoma. Gastroenterology 2005, 129:1375-1383.

54. Radisky DC, Bissell MJ: Matrix metalloproteinase-induced genomic instability. Curr Opin Genet Dev 2006, 16:45-50.

55. Hoshida Y, Villanueva A, Kobayashi M, Peix J, Chiang DY, Camargo A, Gupta S, Moore J, Wrobel MJ, Lerner J: Gene expression in fixed tissues and outcome in hepatocellular carcinoma. N Engl J Med 2008, 359:1995-2004.

56. Kohga K, Takehara T, Tatsumi T, Miyagi T, Ishida H, Ohkawa K, Kanto T, Hiramatsu N, Hayashi N: Anticancer chemotherapy inhibits MHC class I-related chain a ectodomain shedding by downregulating ADAM10 expression in hepatocellular carcinoma. Cancer Res 2009, 69:8050-8057.

57. Yang $C L$, Jiang FQ, Xu F, Jiang GX: ADAM10 overexpression confers resistance to doxorubicin-induced apoptosis in hepatocellular carcinoma. Tumour Biol 2012, 33:1535-1541.

doi:10.1186/1755-1536-6-17

Cite this article as: Rombouts and Carloni: The fibrotic microenvironment as a heterogeneity facet of hepatocellular carcinoma. Fibrogenesis \& Tissue Repair 2013 6:17. 